\title{
Der Einsatz von Virtueller Realität in der Psychotherapeutischen Praxis: Aktueller Forschungsstand, Chancen, Risiken und Herausforderungen
}

\author{
Alla Machulska · Kati Roesmann · Tanja Joan Eiler · Armin Grünewald · Rainer Brück · Tim Klucken
}

Angenommen: 4. November 2021 / Online publiziert: 3. Dezember 2021

(C) Der/die Autor(en) 2021

\begin{abstract}
Zusammenfassung In den letzten Jahren sind durch die Fortschritte der Digitalisierung neue psychotherapeutische Behandlungsmöglichkeiten und Unterstützungsangebote entstanden. Während sich bestimmte Innovationen wie die Videotherapie im letzten Jahr stark verbreitet haben, sind andere Formate wie z. B. Psychotherapieanwendungen in der Virtuellen Realität (VR) zwar sehr gut wissenschaftlich untersucht, aber dennoch kaum in die (tägliche) Praxis implementiert worden.

Der folgende Artikel gibt einen Überblick über den aktuellen Forschungsstand zum Einsatz von VR im Kontext Psychotherapie. Hierbei wird auf zwei wichtige (kognitiv-verhaltenstherapeutische) Interventionsmethoden fokussiert, bei denen VR oft eingesetzt und untersucht wurde: (1) Behaviorale Exposition bei Angsterkrankungen und (2) Modifikation von Informationsverarbeitungsprozessen (Cognitive Bias Modification, CBM). Es werden aktuelle Studien zur Wirksamkeit sowie weitere aktuelle Ergebnisse zur Nutzung und dem Einsatz diskutiert. Weiterhin beschreibt der Artikel die grundsätzliche Nutzung der Virtuellen Realität und definiert wichtige Begriffe und Anwendungen.
\end{abstract}

Die Autoren Alla Machulska und Kati Roesmann teilen sich die Erstautorenschaft.

A. Machulska $(\bowtie) \cdot K$. Roesmann $\cdot$ T. Klucken Klinische Psychologie und Psychotherapie, Universität Siegen, Adolf-Reichwein-Str. 2a, 57076 Siegen, Deutschland Psychotherapeutische Hochschulambulanz der Universität Siegen, Universität Siegen, Siegen, Deutschland alla.machulska@uni-siegen.de

T. J. Eiler · A. Grünewald · R. Brück

Professur für Medizinische Informatik und

Mikrosystementwurf, Universität Siegen, Siegen,

Deutschland
Schlüsselwörter Virtuelle Realität · Wirksamkeit . Exposition · Konfrontation · Cognitive Bias Modification $\cdot$ Immersion

Virtual reality utilization in psychotherapeutic practice: present state of research, opportunities, risks, and challenges

Summary With advances in digitalization, novel psychotherapeutic interventions and supportive programs have evolved in recent years. While certain innovations underwent a wide and rapid dissemination (e.g., video-based consultation), other applications such as virtual-reality-supported psychotherapy are rather seldomly embedded into daily practice despite empirical evidence for their effectiveness.

The present paper provides an overview of the current state of research regarding the use of virtual-reality (VR) in the realm of psychotherapy. In doing so, we will focus on two central (cognitive-behavioral) interventions which were most frequently enriched by VR-based applications in the past: (1) Behavioral exposure in anxiety disorders and (2) modification of information processing (Cognitive Bias Modification, $\mathrm{CBM}$ ). We discuss recent research regarding efficacy of VR-based techniques as well as their utilization and application. Furthermore, the paper describes basic issues of implementation and specifies key terms and applications.

\section{Die Digitalisierung der Psychotherapie}

Psychotherapie unterzieht sich einem ständigen Wandel. Neben inhaltlich-konzeptuellen Weiter- bzw. Neuentwicklungen im Rahmen der 3. Welle hat sich im letzten Jahrzehnt die Psychotherapie durch technologische Innovationen und die Digitalisierung verändert. Digitale Anwendungen im Praxismanagement 
(z.B. elektronische Patientenakte), in der Kommunikation (z. B. Videotherapie), aber auch in psychotherapeutischen Anwendungen (z. B. Einsatz von Virtueller Realität, VR) finden immer mehr Eingang in die psychotherapeutische Versorgung.

Die Auswirkungen der aktuellen Covid19-Pandemie beschleunigten vermutlich den zunehmenden Einfluss digitaler Lösungen in den psychotherapeutischen Alltag. Aktuelle Studien zeigten beispielsweise, dass mittlerweile $75 \%$ aller psychotherapeutischen Praxen Videosprechstunden anbieten (Albrecht et al. 2020).

Im Gegensatz dazu wird der Einsatz neuer Technologien im Rahmen psychotherapeutischer Anwendungen (z.B. Smartphoneapplikationen oder VRAnwendungen) weniger gezielt genutzt. Um Entwicklungen in diesem Bereich zu unterstützen, wird u. a. in Deutschland und Österreich die Verknüpfung von digitalen Lösungen und Psychotherapie politisch zunehmend gefördert. Ende 2019 ist das DigitaleVersorgungs-Gesetz in Deutschland verabschiedet worden: Psychotherapeutische Praxen dürfen seitdem zertifizierte digitale Gesundheitsanwendungen (DiGA) wie Smartphone-Apps „auf Rezept“ verschreiben, wodurch die Nutzung der Anwendungen für Patienten_innen kostenfrei wurde. In Österreich gibt es bislang keine Möglichkeit auf Kostenerstattung durch Kranken- und Gesundheitskassen, da klare und abgestimmte Kriterien zur Beurteilung solcher Anwendungen fehlen. Dennoch sind auch hier ähnliche Entwicklungen $\mathrm{zu}$ verzeichnen: Im Dezember 2020 hat das Austrian Institute for Health Technology Assessment in Form eines Health Technology Assessment-Berichts eine erste Orientierungshilfe zur evidenzbasierten Bewertung von Apps mit Gesundheitsbezug entwickelt, die zukünftig ein einheitliches Vorgehen bei der Evaluation und damit verbunden auch Dissemination und Kostenerstattung sicherstellen könnte. Vor dem Hintergrund dieser Entwicklungen sind professionelle Anwendungen entstanden, die VR im Kontext Psychotherapie explizit nutzen. Es ist daher zu erwarten, dass dezidierte Angebote für

Tab. 1 Abkürzungsverzeichnis

\begin{tabular}{|c|c|c|}
\hline $\begin{array}{l}\text { Abkür- } \\
\text { zung }\end{array}$ & Bedeutung & Erklärung \\
\hline$A p p$ & Applikation & $\begin{array}{l}\text { Anwendungssoftware für Mobilgeräte oder Compu- } \\
\text { ter }\end{array}$ \\
\hline CBM & $\begin{array}{l}\text { Cognitive Bias } \\
\text { Modification }\end{array}$ & $\begin{array}{l}\text { Sammelbegriff für computer-gestützte Verfahren, } \\
\text { die auf eine direkte Veränderung von Informations- } \\
\text { verarbeitungsprozessen mittels Trainings abzielen }\end{array}$ \\
\hline DiGA & $\begin{array}{l}\text { Digitale Ge- } \\
\text { sundheitsan- } \\
\text { wendungen }\end{array}$ & $\begin{array}{l}\text { Medizinprodukte mit gesundheitsbezogener Anwen- } \\
\text { dung, die auf digitalen Technologien basieren und } \\
\text { von deutschen Krankenkassen erstattet werden }\end{array}$ \\
\hline$H M D$ & $\begin{array}{l}\text { Head-Mounted- } \\
\text { Display }\end{array}$ & $\begin{array}{l}\text { Ein auf dem Kopf zu tragendes visuelles Ausgabe- } \\
\text { gerät (meist Brille) }\end{array}$ \\
\hline VE & $\begin{array}{l}\text { Virtual Environ- } \\
\text { ment }\end{array}$ & Vollständige virtuellen Umgebung \\
\hline$V R$ & Virtual Reality & $\begin{array}{l}\text { Computergenerierte interaktive, virtuelle Wirklich- } \\
\text { keit mit Bild (3D) und Ton }\end{array}$ \\
\hline
\end{tabular}

den niedergelassenen psychotherapeutischen Alltag zunehmend entwickelt werden.

Der vorliegende Beitrag verfolgt das Ziel, einen Einblick über den psychotherapeutischen Einsatz von VR zu geben. Einen Überblick über wichtige verwendete Begriffe und Abkürzungen liefert Tab. 1. Es werden zwei Interventionsmethoden hervorgehoben, die in der Forschung besonders intensiv untersucht und evaluiert wurden: (1) VR-basierte Expositionsverfahren bei Angsterkrankungen sowie (2) VR-basierte Verfahren zur Modifikation von Informationsverarbeitungsprozessen (Cognitive Bias Modification, CBM). An dieser Stelle sei angemerkt, dass sich neue Entwicklungen und Forschungen im Kontext digitaler Anwendungen bislang zwar verstärkt auf verhaltenstherapeutische Konzepte stützen, aber zunehmend auch psychodynamische Anwendungen diskutiert werden (Lindegaard et al. 2020). Bis auf einige wenige Fallstudien (Wiederhold et al. 2010) oder psychodynamische Einordnungen von expositionsbasierten VRAnwendungen (Optale et al. 2004) sind uns bisher keine Studien in diesem Bereich bekannt, weswegen sich der Artikel v. a. auf kognitiv-verhaltenstherapeutische VR-Anwendungen bezieht.

\section{Was ist Virtuelle Realität? Begriffsklärungen und praktische Empfehlungen}

Als VR bezeichnet man eine computergenerierte, alternative Realität, in der Nutzer_innen interagieren und sich frei bewegen können (Sherman \& Craig 2003). Durch die Verwendung eines VR-Headsets - eine Art große Brille, die Bildschirme enthält und auch als Head-Mounted-Display (HMD) bezeichnet wird werden Nutzer_innen visuell (und optional auch auditiv) in die VR „transportiert“. Die Einsatzzwecke einer vollständig virtuellen Umgebung (engl. Virtual Environment, VE) sind vielfältig und reichen von medizinischen und psychologischen Anwendungsbereichen (z.B. OP-Simulationen, Therapie von Ängsten) über den Bereich der (Fort-)Bildung allgemein, bis hin zur Unterhaltungsindustrie. VR unterscheidet sich u.a. durch die Phänomene Immersion und Präsenz von anderen Mensch-Computer-Interaktionen (Riva 2005). Immersion beschreibt das „Eintauchen“ in eine andere Welt und kann durch die Erzeugung unterschiedlicher Sinneseindrücke erreicht werden. Als Präsenzerleben bezeichnet man den Eindruck des Nutzers/der Nutzerin, tatsächlich in einer anderen Welt $\mathrm{zu}$ sein und nicht nur computergenerierte Bilder zu sehen. Dieses psychologische Phänomen spiegelt sich u. a. darin wider, dass Nutzer_innen eher auf Ereignisse in der virtuellen als in der realen Welt reagieren. VR ermöglicht es also, die virtuelle Umgebung als real und unvermittelt wahrzunehmen, trotz des Wissens, dass es sich um eine computergenerierte Welt handelt (Lee 2004).

Ein hohes $\mathrm{Maß}$ an Immersion wird durch einen natürlich wirkenden Sichtbereich (z. B. kein sichtba- 
res Ende der virtuellen Welt), die Realisierung von Tiefeneindruck durch stereoskopisches Sehen, sowie durch realistisch wirkende Interaktionen mit Objekten erzielt. Im Idealfall werden neben der visuellen Modalität auch weitere Modalitäten (Hören, Schmecken, Riechen, Tasten) angesprochen (Beier 2003).

Das (in der Regel) kabelgebundene, stationäre High-end VR-System ist die qualitativ, aber auch preislich höchstwertige VR-Variante. Hierbei wird ein HMD mit einem leistungsstarken PC verbunden, der die Bild- und Tondaten für das HMD bereitstellt. Das HMD sowie optional auch Eingabegeräte (Controller) werden über ein externes System „getrackt“, das heißt, ihre Position im Raum wird ermittelt. Vorteile dieses Systems sind die sehr gute Bild- und „Tracking“-Qualität. Nachteile sind die hohen Anschaffungskosten (ca. 800-1200€ plus PC) sowie eine eingeschränkte Bewegungsfreiheit durch das Kabel.

Standalone VR-Systeme, auch als all-in-one-HMD bezeichnet, sind im Gegensatz dazu weder auf einen externen PC angewiesen, noch auf ein externes Trackingsystem. Stattdessen befinden sich in dem HMD ein Prozessor, sowie diverse Sensoren und Kameras, welche die Kopfbewegung erfassen und den Raum um das HMD herum abtasten, um so etwa Controller oder auch die eigenen Hände zu erkennen. Vorteile dieser Systeme sind die geringeren Anschaffungskosten (ca. 350-500€) und große Bewegungsfreiheit. Nachteile sind das schwächere Tracking sowie die geringere graphische Qualität.

Bei Smartphone-basierten VR-Systemen wird das Smartphone in eine dafür vorgesehene Halterung gelegt und eine App gestartet, die eine stereoskopische Ansicht unterstützt. Dazu werden - wie bei anderen HMDs auch - zwei einzelne Bilder auf die Augen gelegt. Mit Hilfe der in dem Smartphone verbauten Sensoren kann zudem die Drehung des Kopfes an die App übertragen werden. Auf Grund der fehlenden Tracking-Möglichkeit und der somit nicht vorhandenen Orientierung im Raum eignet es sich insbesondere für sitzende Interaktionen wie etwa $360^{\circ}$ Videos. Vorteile dieses Systems sind die geringen Kosten (geeignete Smartphone-Halterungen gibt es schon ab ca. $30 €)$, Nachteile sind die eingeschränkte Nutzbarkeit (Harley et al. 2019).

\section{Chancen von VR in der Psychotherapie}

Die Möglichkeit virtuelle Umgebungen flexibel gestalten und verändern zu können bietet für die Psychotherapie ein hohes Potenzial. Aufgrund ihrer immersiven Eigenschaft eignet sich VR, um störungs- und therapierelevante Situationen alltagsnah $\mathrm{zu}$ simulieren und um Person-Umwelt-Interaktionen im virtuellen Raum gezielt zu ermöglichen. So können individuelle Lerngelegenheiten geschaffen werden, in denen Patienten_innen Verhaltensalternativen erproben können. Dabei ist VR mit einer hohen Motivation der Nutzer_innen verbunden (Forman et al. 2018). Gleich- zeitig bietet die Arbeit mit VR ein Höchstmaß an experimenteller Kontrolle und Vorhersagbarkeit - ein Aspekt, der nicht nur für die Gestaltung der Lernsituationen, sondern auch für die psychotherapeutische Wirksamkeits- und Prozessforschung von hohem Nutzen ist.

Trotz dieser Vorteile möchten wir vorab kritisch anmerken, dass viele der im Folgenden genannten Studien zum Einsatz und zur Wirksamkeit von VR in der Psychotherapie aufgrund niedriger Fallzahlen, fehlender Kontrollgruppen sowie fehlender Daten zu langfristigen Therapieeffekten nur vorläufige Aussagen über den tatsächlichen Nutzen VR-basierter Interventionen zulassen. Dies betrifft insbesondere das Anwendungsfeld CBM (s. unten). Für eine abschließende Beurteilung des klinischen Nutzens von $\mathrm{VR}$ in den nachfolgend skizzierten Anwendungsbereichen sind (weitere) randomisiert-kontrollierte Studien mit konventionellen Therapien als aktive Kontrollbedingung und ausreichender Stichprobengröße wünschenswert.

\section{Einsatz von Virtueller Realität in Expositionstherapien bei Angsterkrankungen}

Virtuelle Realitäten werden im Kontext der Behandlung von Angsterkrankungen seit den 90er-Jahren zunehmend untersucht (Morina et al. 2015). Angststörungen stellen mit einer geschätzten 12-Monatsprävalenz von ca. $15 \%$ eine der größten Gruppen psychischer Störungen dar (Wittchen et al. 2011). Bei Betroffenen führt exzessives Furcht- und Vermeidungsverhalten zu mitunter drastischen Einschränkungen im Lebensalltag sowie zu einem erhöhten Risiko, weitere komorbide psychische Erkrankungen zu entwickeln (Wardenaar et al. 2017). Die Expositionstherapie ist mit mittleren bis hohen Effektstärken die Therapie der Wahl für die Behandlung von Angststörungen (Bandelow et al. 2014). Dabei konfrontieren sich Angstpatienten_innen unter therapeutischer Anleitung und im Verlauf zunehmend selbstgesteuert systematisch mit individuell angstauslösenden Situationen, welche im Alltag vermieden werden, und können dadurch ihre Befürchtungen überprüfen und einen neuen Umgang mit der Angst lernen.

In mehreren Metaanalysen und systematischen Reviews konnte gezeigt werden, dass in virtuo Expositionstherapien vergleichbare Effektstärken erzielen wie traditionelle, therapeutengeleitete in vivo Expositionen (Carl et al. 2019), und dass ein Transfer der Lernerfahrungen in das „Real-Life“ stattfindet (Morina et al. 2015).

Am besten untersucht ist die Effektivität von in virtuo Expositionen bei spezifischen Phobien, wie der Flug- (Cardoş et al. 2017; Campos et al. 2019), der Höhen- (Donker et al. 2019) oder der Tierphobie (Leehr et al. 2021; Suso-Ribera et al. 2019). Dies ist sicherlich einerseits dadurch erklärbar, dass phobische Reize/ Situationen bei spezifischen Phobien vergleichsweise 
konkret und wenig komplex sind und entsprechende VR-Szenarien somit relativ einfach kreiert werden können. Andererseits nimmt bei komplexeren Angsterkrankungen im Vergleich zur spezifischen Phobie die kognitive Arbeit (z.B. Veränderung von „Selbstaufmerksamkeit" auf eigene körperliche Veränderungen und Überprüfung dysfunktionaler Schemata bei Sozialer Phobie) einen zentraleren Stellenwert in der Therapie ein, wodurch möglicherweise der alleinige Effekt der in virtuo Expositionen geringer ausfallen könnte. Nichtsdestotrotz gelten diese auch bei komplexeren Phobien wie der Sozialen Phobie (Horigome et al. 2020) und der Agoraphobie (Meyerbroeker et al. 2013) als effektiv. In Wirksamkeitsvergleichen finden sich in Abhängigkeit spezifischer Studiencharakteristika (Wechsler et al. 2019) sowie des Messzeitpunkts von Therapieerfolg (Horigome et al. 2020) teilweise Hinweise auf eine Überlegenheit von in vivo gegenüber in virtuo Verfahren (insbesondere für die Soziale Phobie). Möglicherweise ist diese Varianz dadurch erklärbar, dass Variablen, welche den kurz- und langfristigen Erfolg von expositions-basierten Interventionen bedingen (z.B. Kontextvariation, Erwartungsverletzungen, Kontrolle von Sicherheitsverhalten, Dauer der Exposition, etc.; vgl. Böhnlein et al. 2020; Craske et al. 2018), in unterschiedlichem Grade in die Konzeption der klinischen Studien eingeflossen sind. Um das Potenzial virtueller Realitäten voll ausschöpfen und Applikationen evidenzbasiert weiter optimieren zu können, ist es daher wichtig, die spezifischen Wirkmechanismen von in virtuo Expositionen (Wechsler et al. 2019) wie auch allgemeine Marker für Therapieerfolg von Expositionstherapien (Böhnlein et al. 2020) gezielt zu untersuchen und - soweit möglich - in die Konstruktion individualisierter Applikationen einfließen zu lassen.

Insgesamt hat sich mit zunehmenden Wirksamkeitsnachweisen auch die Haltung der Kliniker_innen gegenüber in virtuo Expositionen in eine positive Richtung verschoben (Anderson und Molloy 2020). Dennoch haben diese in die ambulante klinische Versorgung bislang kaum Einzug gehalten. Eine zukünftig stärkere Dissemination von VR-Applikationen könnte jedoch gerade für niedergelassene Therapeuten_innen die Durchführung von Expositionen deutlich erleichtern, da Patienten_innen unter therapeutischer Begleitung direkt in der Praxis mit relevanten, angstassoziierten Situationen/Reizen konfrontiert werden könnten. Dadurch könnten sowohl für Patienten_innen, wie auch für Therapeuten_innen (ökonomisch unattraktive) Zeiten für die An- und Abreise zum Ort der geplanten Expositionsübung reduziert oder sogar vermieden werden. Internetbasierte VRApplikationen ermöglichen sogar vollständig selbstgesteuerte Expositionsübungen ohne therapeutische Anleitung, wobei es für Patient_innen mit spezifischen Phobien erste Evidenz für die Effektivität dieses therapeutenunabhängigen Einsatzes gibt (Donker et al. 2019; Campos et al. 2019). Weiterhin erlauben VR-
Applikationen ein wiederholtes Üben in multiplen Kontexten und mit unterschiedlichen Angstreizen, welches die Generalisierung von Erfahrungen in den Alltag der Patienten_innen erleichtern und Rückfällen vorbeugen könnte (de Jong et al. 2019). Eine weitere Hoffnung ist es, dass durch VR-Angebote auch Patienten_innen erreicht werden könnten, die sich - zum Beispiel aufgrund eines besonders ausgeprägten Vermeidungsverhaltens - auf eine traditionelle Expositionsbehandlung nicht einlassen würden.

\section{Einsatz von Virtueller Realität bei Cognitive Bias Modification}

Der Umstand, dass Menschen ein „subjektives“ Abbild der äußeren Welt erzeugen, welches Verzerrungen unterliegen kann, ist seit Jahrzehnten Gegenstand der psychologischen Forschung und psychotherapeutischen Arbeit ( $\mathrm{zu}$ positiven Verzerrungen bzw. Illusionen s. z. B. Taylor (1989); einen Überblick über Verzerrungen der Informationsverarbeitung im Kontext psychischer Störungen geben Wiers et al. (2007)). Cognitive Bias Modification (CBM) umfasst eine Reihe von Verfahren, die sich zunächst aus der Grundlagenforschung heraus entwickelt haben und nun zunehmend in die therapeutische Praxis Einzug halten. Hintergrund dieses Ansatzes ist, dass maladaptive Verzerrungen der Informationsverarbeitung - sog. „cognitive biases“ (z. B. Aufmerksamkeitsfokus auf Bedrohungsreize im Kontext von Phobien; veränderte Assoziationen und automatische Annäherungsoder Vermeidungsverhaltensweisen) - an der Entstehung und Aufrechterhaltung verschiedener psychischer Störungen beteiligt sind, sodass hier von einem transdiagnostischen Faktor gesprochen werden kann (Wiers et al. 2013). Gleichzeitig scheinen solche Prozesse änderungssensitiv zu sein und nehmen nach erfolgreicher Psychotherapie ab (Neimeijer et al. 2015). CBM zielt auf eine direkte Veränderung dieser Prozesse durch computergestützte Trainingsverfahren ab, um so zur Symptomreduktion beizutragen (BarHaim 2010). Ein herausragendes Beispiel für eine gelungene und effektive Translation von der Forschung in die Praxis stellt das Joysticktraining im Kontext der Rückfallprophylaxe bei Alkoholabhängigkeit dar (Loijen et al. 2020). Hier lernen Personen, auf alkohol-bezogene Bilder automatisch mit einer Vermeidungsbewegung zu reagieren, während alkoholfreie Getränke mittels eines Joysticks angenähert werden. In mehreren Studien konnte gezeigt werden, dass 4-6 Sitzungen à 15 min zu Beginn einer stationären Entwöhnungstherapie nicht nur alkoholbezogene Informationsverarbeitungsprozesse verändern, sondern auch die Rückfallraten nach einem Jahr um ca. 10\% senken (z. B. Wiers et al. 2011). Daher ist das JoystickTraining gegen Alkohol in einigen Kliniken und RehaZentren in Deutschland zu einem zusätzlichen Baustein des Therapieangebots geworden (z.B.: http:// www.salus-materialien.de/). 
Obwohl CBM-Trainings theoretisch fundiert, experimentell gut untersucht und in einigen Störungsbereichen vielversprechend sind, sind die Befunde nicht immer eindeutig (z. B. Boffo et al. 2019). Dabei stellen die oftmals kleinen Effektstärken und die eingeschränkte Generalisierung auf alltägliches Verhalten die größten Einschränkungen dar. Die Darbietung solcher Verfahren in der VR könnte eine vielversprechende Möglichkeit darstellen, auf diese Grenzen zu reagieren. Tatsächlich wurden in der jüngsten Zeit einige Versuche unternommen, CBM um VR zu ergänzen. So haben Urech und Kollegen (2015) ein Aufmerksamkeitstraining in der VR entwickelt, bei dem sozial ängstliche Personen ihre Aufmerksamkeit von bedrohungsassoziierten (negativer emotionaler Gesichtsausdruck) hin zu neutralen Reizen (neutrale Gesichtsausdrücke) lenken sollten. Neben einer hohen Akzeptanz des Trainings zeigten sich auch Veränderungen der Aufmerksamkeit (reduzierte Aufmerksamkeit für Bedrohungsreize) und der selbstberichteten sozialen Ängstlichkeit. In einer anderen Studie von Otkhmezuri und Kollegen (2019) lernten sozial ängstliche Studierende, auf virtuelle ambige Leistungssituationen mit einer positiven Interpretation zu antworten. Im Vergleich zu einem herkömmlichen Computertraining war das VR-Training mit mehr Immersion, einer größeren Reduktion selbstberichteter Ängstlichkeit und geringerer Reaktion auf einen experimentellen Stressor verbunden. Interessanterweise korrelierten diese positiven Verbesserungen mit Gefühlen von Immersion und Präsenz, was für den inkrementellen Nutzen der VR spricht.

Trotz vielversprechender Ergebnisse ist bei den obigen Studien einschränkend zu erwähnen, dass es sich um Pilotstudien mit kleinen und subklinischen Stichproben handelt, sodass unklar bleibt, inwiefern sich die Ergebnisse auf Klienten_innen mit einem deutlichen Leidensdruck und Behandlungswunsch übertragen lassen. Eine neuere Studie aus unserer Arbeitsgruppe konnte erste Hinweise dafür liefern, dass CBM in der VR auch im klinischen Kontext erfolgreich durchführbar ist (Machulska et al. 2021). Bei der Studie führten $>100$ zur Abstinenz motivierte Raucher_innen ein zwei-wöchiges Nikotin-Vermeidungstraining in der VR durch, das an das oben beschriebene Alkohol-Joysticktraining angelehnt war. Nach der VR-Intervention konnte der Tabakkonsum um ca. $50 \%$ reduziert werden, was auch in einer 3-Monats-Katamnese bestätigt wurde. Diese Befunde liefern also erste Hinweise für das Potenzial von VR im Kontext CBM.

\section{Mögliche Risiken von VR-Therapien}

Neben den oben beschriebenen Chancen, die durch VR-Anwendung in der Psychotherapie entstehen, möchten wir hier auch auf einige potenzielle Risiken und/oder Nebenwirkungen aufmerksam machen. Hierbei sei jedoch anzumerken, dass systematische
Untersuchungen in diesem Bereich häufig fehlen, sodass dieser Abschnitt eher die in der Literatur vorhandenen Diskussionen und Bedenken beleuchtet.

Die häufigsten Kritikpunkte beziehen sich auf Bedenken bezüglich der Akzeptanz von VR-Therapien seitens Betroffener, mögliche unerwünschte Einflüsse auf die therapeutische Allianz und die oftmals unzureichende Individualisierung der VR-Szenarien, einhergehend mit der fehlenden Berücksichtigung des soziokulturellen Lebensraums von Klienten_innen (vgl. Emmelkamp und Meyerbröker 2021). Dies wiederum kann Einfluss auf Therapieabbrüche und mögliche Symptomverschlechterung haben.

Tatsächlich zeigen erste Studien, dass Klient_innen Expositionen in vivo VR-gestützten Expositionen nicht vorziehen (Garcia-Palacios et al. 2007) und auch die therapeutische Beziehung bei beiden Vorgehen vergleichbar ist (Ngai et al. 2015). Dennoch ist anzunehmen, dass durch den zunehmenden Einsatz von digitalen Technologien der therapeutische Prozess und die therapeutische Beziehung verändert werden. Durch den Gebrauch von digitalen Technologien rückt ein zusätzlicher Faktor in den Gesprächsfokus, sodass es ggf. zu einer triadischen (Therapeut_in-VR-Klient_in) Situation kommt. Ein Teil der therapeutischen Gespräche wird sich zwangsläufig mit dem Gebrauch, Einsatz, den Problemen und Wirkungen der digitalen Technologien beschäftigen. Zudem ändern sich die Therapeut_in-Klient_in-Interaktion, da z. B. Augenkontakt und andere ermutigende Gesten während der Verwendung von VR-Applikationen nicht stattfinden. Inwiefern sich dies auf andere wichtige Prozesse auswirkt wie z.B. Beziehung, Allianz, Erfolgserwartung oder Übertragung ist bislang noch nicht untersucht.

Ein durchaus valider Kritikpunkt betrifft zudem die eingeschränkte (soziokulturelle) Berücksichtigung des individuellen Kontextes. Hier gibt es Hinweise darauf, dass die Therapiewirksamkeit durch die Programmierung passender individueller virtueller Kontexte und Szenarien verbessert werden kann (Garcia-Batista et al. 2020), was jedoch mit einem hohen Zeitaufwand und ökonomischen Einsatz verbunden ist.

Was Drop-out und Symptomverschlechterung angeht, zeigen bisherige Studien erfreulicherweise keinen signifikanten Unterschied zu herkömmlicher Therapie (für einen umfassenden Überblick sei auf Emmelkamp und Meyerbröker 2021 verwiesen).

\section{Herausforderungen: Transfer von VR- Applikationen aus dem Forschungslabor in die klinische Versorgung}

Der Einsatz von VR wurde und wird im Kontext der Psychotherapie viel beforscht. In einem umfassenden, aktuellen Review arbeiteten Emmelkamp und Meyerbröker (2021) neben den hier beschriebenen Störungsbereichen auch für weitere psychische Störungen die Befunde systematisch auf: Über den Einsatz von VR im Kontext der expositions-basierten 
Therapie von (phobischen) Angststörungen hinaus gebe es zunehmend Evidenz für einen wirksamen Einsatz bei Suchterkrankungen und Essstörungen. Evidenz im Bereich der Zwangserkrankungen und der generalisierten Angststörung sei begrenzt. Weiterhin erscheine laut Emmelkamp und Meyerbröker (2021) der therapeutische Einsatz von VR bei psychotischen und Autismusspektrumsstörungen sowie Aufmerksamkeitsdefizit- und Hyperaktivitätsstörungen vielversprechend.

Trotz - für einige Störungsbereiche - vielversprechender Forschungsbefunde ist jedoch der Transfer von VR in die psychotherapeutische Praxis bislang begrenzt. Für Therapeuten_innen kann es herausfordernd sein, serienmäßig produzierte VR-Programme zu finden, welche auf die speziellen Bedürfnisse ihrer Patienten_innen abgestimmt und handhabbar sind. Eine Herausforderung für die kommenden Jahre wird daher die Verbesserung der Benutzerfreundlichkeit und damit auch die vereinfachte Anpassung von VRSzenarien und -elementen durch den Anwender/die Anwenderin (Praktiker_in und/oder Klient_in) sein, um das Potenzial, welches VR bietet (z. B. Kontextvariation und Alltagsnähe zu Förderung von Transfereffekten), voll ausschöpfen zu können. Allgemein ist positiv zu vermerken, dass die VR-Technologie sowie die Entwicklung therapierelevanter Software in den vergangenen Jahren erhebliche Fortschritte gemacht haben, sodass vollständige VR-Systeme für niedergelassene Therapeuten_innen immer bezahlbarer werden und dadurch zukünftig mehr in der Routineversorgung Einzug halten könnten.

Funding Open Access funding enabled and organized by Projekt DEAL.

Interessenkonflikt A. Machulska, K. Roesmann, T.J. Eiler, A. Grünewald, R. Brück und T. Klucken geben an, dass kein Interessenkonflikt besteht.

Open Access Dieser Artikel wird unter der Creative Commons Namensnennung 4.0 International Lizenz veröffentlicht, welche die Nutzung, Vervielfältigung, Bearbeitung, Verbreitung und Wiedergabe in jeglichem Medium und Format erlaubt, sofern Sie den/die ursprünglichen Autor(en) und die Quelle ordnungsgemäß nennen, einen Link zur Creative Commons Lizenz beifügen und angeben, ob Änderungen vorgenommen wurden.

Die in diesem Artikel enthaltenen Bilder und sonstiges Drittmaterial unterliegen ebenfalls der genannten Creative Commons Lizenz, sofern sich aus der Abbildungslegende nichts anderes ergibt. Sofern das betreffende Material nicht unter der genannten Creative Commons Lizenz steht und die betreffende Handlung nicht nach gesetzlichen Vorschriften erlaubt ist, ist für die oben aufgeführten Weiterverwendungen des Materials die Einwilligung des jeweiligen Rechteinhabers einzuholen.

Weitere Details zur Lizenz entnehmen Sie bitte der Lizenzinformation auf http://creativecommons.org/licenses/by/4. 0/deed.de.

\section{Referenzen}

Albrecht, M., Otten, M., Sander, M., \& Temizdemir, E. (2020). PraxisBarometer Digitalisierung 2020. Ergebnisbericht für die Kassenärztliche Bundesvereinigung. https: / /www. $\mathrm{kbv.de/html/praxisbarometer.php.} \mathrm{Zugegriffen:} \mathrm{29.} \mathrm{Apr.}$ 2021.

Anderson, P.L., \& Molloy, A. (2020). Maximizing the impact of virtual reality exposure therapy for anxiety disorders. Current opinion in psychology, 36, 153-157.

Bandelow, B., Wiltink, J., Alpers, G. W., Benecke, C., Deckert, J., Eckhardt-Henn, A., Ehrig, C., Engel, E., Falkai, P., Geiser, F., Gerlach, A.L., Harfst, T., Hau, S., Joraschky, P., Kellner, M., Köllner, V., Kopp, I., Langs, G., Lichte, T., Liebeck, H., Matzat, J., Reitt, M., Rüddel, H. P., Rudolf, S., Schick, G., Schweiger, U., Simon, R., Springer, A., Staats, H., Ströhle, A., Ströhm, W., Waldherr, B., Watzke, B., Wedekind, D., Zottl, C., Zwanzger, P., Beutel M.E. (2014). Deutsche S3Leitlinie Behandlung von Angststörungen

Bar-Haim, Y. (2010). Research review: Attention bias modification (ABM): a novel treatment for anxiety disorders. Journal of child psychology and psychiatry, and allied disciplines, 51, 859-870.

Beier, K.-P. (2003). Virtual reality: a short introduction. http:// www.umich.edu/ vrl/intro/.Zugegriffen: 1. Mai2021.

Boffo, M., Zerhouni, O., Gronau, Q. F., van Beek, R. J. J., Nikolaou, K., Marsman, M., \& Wiers, R.W. (2019). Cognitive bias modification for behavior change in alcohol and smoking addiction: Bayesian meta-analysis of individual participant data. Neuropsychology Review, 29, 52-78.

Böhnlein, J.,Altegoer, L., Muck, N. K., Roesmann, K., Redlich, R., Dannlowski, U., \& Leehr, E. J. (2020). Factors influencing the success of exposure therapy for specific phobia: A systematic review. Neuroscience and Biobehavioral Reviews, 108, 796-820.

Campos, D., Bretón-López, J., Botella, C., Mira, A., Castilla, D., Mor, S., Baños, R., \& Quero, S. (2019). Efficacy of an internet-based exposure treatment for flying phobia (NOFEAR airlines) with and without therapist guidance: a randomized controlled trial. BMC Psychiatry, 19(1), 86. https:// doi.org/10.1186/s12888-019-2060-4.

Cardoş, R. A. I., David, O. A., \&David, D. O. (2017).Virtual reality exposure therapy in flight anxiety: a quantitative metaanalysis. Computers in Human Behavior, 72, 371-380.

Carl, E., Stein, A.T., Levihn-Coon, A., Pogue, J. R., Rothbaum, B., Emmelkamp, P., Asmundson, G. J.G., Carlbring, P., \& Powers, M. B. (2019). Virtual reality exposure therapy for anxiety and related disorders: a meta-analysis of randomized controlled trials. Journal of Anxiety Disorders, 61,27-36.

Craske, M. G., Hermans, D., \& Vervliet, B. (2018). State-ofthe-art and future directions for extinction as a translational model for fear and anxiety. Philosophical Transactions of the Royal Society B: Biological Sciences, 373(1742), 20170025.https://doi.org/10.1098/rstb.2017.0025.

Donker, T., Cornelisz, I., Van Klaveren, C., Van Straten, A., Carlbring, P., Cuijpers, P., \& Van Gelder, J. L. (2019). Effectiveness of self-guided app-based virtual reality cognitive behavior therapy for acrophobia: a randomized clinical trial. JAMA Psychiatry, 76(7), 682-690.

Emmelkamp, P.M.G., \& Meyerbröker, K. (2021). Virtual reality therapy in mental health. Annual Review of Clinical Psychology, 17, 495-519.

Forman, E. M., Goldstein, S. P., Flack, D., Evans, B. C., Manasse, S. M., \& Dochat, C. (2018). Promising technological innovations in cognitive training to treat eating-related behavior. Appetite, 24,68-77. 
Garcia-Batista, E. Z., Guerra-Pena, K., Alsina-Jurnet, I., CanoVindel, A., Herrera Martinez, S.X., et al. (2020). Design of virtual environments for the treatment of agoraphobia: inclusion of culturally relevant elements for the population of the Dominican Republic. Comput Hum Behav, 102, 97-102.

Garcia-Palacios, A., Botella, C., Hoffman, H., \& Fabregat, S. (2007). Comparing acceptance and refusal rates of virtual reality exposure versus in vivo exposure by patients with specific phobias. Cyberpsychol Behav, 10, 722-724.

Harley, D., Tarun, A. P., Elsharawy, S., Verni, A., Tibu, T., Bilic, M., Bakogeorge, A., \& Mazalek, A. (2019on). Mobile Realities. In S. Harrison, S. Bardzell, C. Neustaedter \& D. Tatar (Hrsg.), Proceedings of the 2019 on designing interactive systems conference (S. 1131-1144).:ACM.

Horigome, T., Kurokawa, S., Sawada, K., Kudo, S., Shiga, K., Mimura, M., \& Kishimoto, T. (2020). Virtual reality exposure therapy for social anxiety disorder: a systematic review and meta-analysis. Psychological Medicine, 50(15), 2487-2497.

deJong, R., Lommen, M. J. J., de Jong, P. J., \& Nauta, M. H. (2019). Using multiple contexts and retrieval cues in exposurebased therapy to prevent relapse in anxiety disorders. Cognitive and Behavioral Practice, 26(1), 154-165.

Lee, K. M. (2004). Presence, explicated. Communication Theo$r y, 14(1), 27-50$.

Leehr, E. J., Roesmann, K., Böhnlein, J., Dannlowski, U., Gathmann, B., Herrmann, M. J., Junghöfer, M., Schwarzmeier, H., Seeger, F. R., Siminski, N., Straube, T., Lueken, U., \& Hilbert, K. (2021). Clinical predictors of treatment response towards exposure therapy in virtuo in spider phobia: a machine learning and external cross-validation approach. Journal of Anxiety Disorders, 83, 102448. https://doi.org/ 10.1016/j.janxdis.2021.102448.

Lindegaard, T., Hesslow, T., Nilsson, M., Johansson, R., Carlbring, P., Liliengren, P., \& Andersson, G. (2020). Internetbased psychodynamic therapy vs cognitive behavioural therapy for social anxiety disorder: a preference study. Internet Interventions, 20, 100316.

Loijen, A., Vrijsen, J.N., Egger, J., Becker, E.S., \& Rinck, M. (2020). Biased approach-avoidance tendencies in psychopathology: a systematic review of their assessment and modification. Clinical psychology review, 77, 101825.

Machulska, A., Eiler, T. J., Kleinke, K., Grünewald, A., Brück, R., Jahn, K., Niehaves, B., \& Klucken, T. (2021). Approach bias retraining through virtual reality in smokers willing to quit smoking: arandomized control study. Behaviour Research and Therapy, 141, 103858.

Meyerbroeker, K., Morina, N., Kerkhof, G. A., \& Emmelkamp, P.M. G. (2013). Virtual reality exposure therapy does not provide any additional value in agoraphobic patients: a randomized controlled trial. Psychotherapy and Psychosomatics, 82(3), 170-176.

Morina, N., Ijntema, H., Meyerbröker, K., \& Emmelkamp, P. M. G. (2015). Can virtual reality exposure therapy gains be generalized to real-life? A meta-analysis of studies applying behavioral assessments. Behaviour Research and Therapy, 74, 18-24.

Neimeijer, R.A., de Jong, P.J., \& Roefs, A. (2015). Automatic approach/avoidance tendencies towards food and the course of anorexia nervosa. Appetite, 91,28-34.

Ngai, I., Tully, E.C., \& Anderson, P.L. (2015). The course of the working alliance during virtual reality and exposure group therapy for social anxiety disorder. Behav Cogn Psychother, 43, 167-181.
Optale, G., Pastore, M., Marin, S., Bordin, D., Nasta, A., \& Pianon, C. (2004). Male sexual dysfunctions: immersive virtual reality and multimedia therapy. Studies in Health Technology and Informatics, 99, 165-178.

Otkhmezuri, B., Boffo, M., Siriaraya, P., Panote Siriaraya, Matsangidou, M., Wiers, R. W., Mackintosh, B., Ang, C. S., \& Salemink, E. (2019). Believing is seeing: a proof-of-concept semiexperimental study on using mobile virtual reality to boost the effects of interpretation bias modification for anxiety. JMIR Ment Health, 6, e11517.

Riva, G. (2005). Virtual reality in psychotherapy: review. Cyberpsychology \& behavior, 8(3), 220-230. discussion 231-40.

Sherman, W. R., \& Craig, A. B. (2003). Understanding virtual reality: interface, application, and design. Morgan Kaufmann series in computer graphics and geometric modeling. : Morgan Kaufmann.

Suso-Ribera, C., Fernández-Álvarez, J., García-Palacios, A., Hoffman, H. G., Bretón-López, J., Baños, R. M., Quero, S., \& Botella, C. (2019). Virtual reality, augmented reality, and in vivo exposure therapy: a preliminary comparison of treatment efficacy in small animal phobia. Cyberpsychology, Behavior, and Social Networking, 22(1), 31-38.

Taylor, S. E. (1989). Positive illusions. NewYork: Basic Books.

Urech, A., Krieger, T., Chesham, A., Mast, F., \& Berger, T. (2015). Virtual reality-based attention bias modification training for social anxiety: a feasibility and proof of concept study. Front Psychiatry, 6, 154.

Wardenaar, K. J., Lim, C.C.W., Al-Hamzawi, A. O., Alonso, J., Andrade, L.H., Benjet, C., Bunting, B., de Girolamo, G., Demyttenaere, K., Florescu, S.E., Gureje, O., Hisateru, T., Hu, C., Huang, Y., Karam, E., Kiejna, A., Lepine, J.P., Navarro-Mateu, F, Oakley Browne, M., Piazza, M., PosadaVilla, J., Ten Have, M. L., Torres, Y., Xavier, M., Zarkov, Z., Kessler, R. C., Scott, K. M., \& de Jonge, P. (2017). The crossnational epidemiology of specific phobia in the World Mental Health Surveys. Psychological Medicine, 47(10), 1744-1760.

Wechsler, T. F., Mühlberger, A., \& Kümpers, F. (2019). Inferiority or even superiority of virtual reality exposure therapy in phobias?-A systematic review and quantitative meta-analysis on randomized controlled trials specifically comparing the efficacy of virtual reality exposure to gold standard in vivo exposure in agoraphobia, specific phobia, and social phobia. Frontiers in Psychology, 10, 1758. https://doi.org/10.3389/fpsyg.2019.01758.

Wiederhold, B. K., Gavshon, L., \& Wiederhold, M. D. (2010). A psychodynamic view of virtual reality exposure therapy. Journal of CyberTherapy and Rehabilitation, 3, 395-403.

Wiers, R. W., Eberl, C., Rinck, M., Becker, E. S., \& Lindenmeyer, J. (2011). Retraining automatic action tendencies changes alcoholicpatients' approachbiasforalcoholandimproves treatment outcome. Psychological Science, 22, 490-497.

Wiers, R.W., Gladwin, T.E., Hofmann, W., Salemink, E., \& Ridderinkhof, K. R. (2013). Cognitive bias modification and cognitive control training in addiction and related psychopathology: Mechanisms, clinical perspectives, and ways forward. Clinical Psychological Science, 1, 192-212.

Wiers, R. W., Teachman, B. A., \& De Houwer, J. (2007). Implicit cognitive processes in psychopathology: an introduction. Journal of behavior therapy and experimental psychiatry, $38,95-104$.

Wittchen, H. U., Jacobi, F., Rehm, J., Gustavsson, A., Svensson, M., Jönsson, B., Olesen, J., Allgulander, C., Alonso, J., Faravelli, C., Fratiglioni, L., Jennum, P., Lieb, R., Maercker, A., Van Os, J., Preisig, M., Salvador-Carulla, L., Simon, R., 


\section{übersichtsarbeit}

\& Steinhausen, H.-C.C. (2011). The size and burden of mental disorders and other disorders of the brain in Europe 2010. European Neuropsychopharmacology, 21(9), 655-679.

Hinweis des Verlags Der Verlag bleibt in Hinblick auf geografische Zuordnungen und Gebietsbezeichnungen in veröffentlichten Karten und Institutsadressen neutral. 